The Astrophysical Journal, 671:802-810, 2007 December 10

(C) 2007. The American Astronomical Society. All rights reserved. Printed in U.S.A.

\title{
CAN EXTRA MIXING IN RGB AND AGB STARS BE ATTRIBUTED TO MAGNETIC MECHANISMS?
}

\author{
Maurizio Busso, ${ }^{1}$ Gerald J. Wasserburg, ${ }^{2}$ Kenneth M. Nollett ${ }^{3}$ and Andrea Calandra ${ }^{1}$ \\ Received 2007 May 31; accepted 2007 August 20
}

\begin{abstract}
It is known that there must be some weak form of transport (called cool bottom processing, or CBP) acting in lowmass red giant branch (RGB) and asymptotic giant branch (AGB) stars, adding nuclei, newly produced near the hydrogen-burning shell, to the convective envelope. We assume that this extra mixing originates in a stellar dynamo operated by the differential rotation below the envelope, maintaining toroidal magnetic fields near the hydrogen-burning shell. We use a phenomenological approach to the buoyancy of magnetic flux tubes, assuming that they induce matter circulation as needed by CBP models. This establishes requirements on the fields necessary to transport material from zones where some nuclear burning takes place, through the radiative layer and into the convective envelope. Magnetic field strengths are determined by the transport rates needed by CBP for the model stellar structure of a star of initially $1.5 M_{\odot}$, in both the AGB and RGB phases. The field required for the AGB star in the processing zone is $B_{0} \sim 5 \times 10^{6} \mathrm{G}$; at the base of the convective envelope this yields an intensity $B_{E} \lesssim 10^{4} \mathrm{G}$. For the RGB case, $B_{0} \sim 5 \times 10^{4}-4 \times 10^{5} \mathrm{G}$, and the corresponding $B_{E}$ are $\sim 450-3500 \mathrm{G}$. These results are consistent with existing observations on AGB stars. They also hint at the basis for high field sources in some planetary nebulae, and the very large fields found in some white dwarfs. It is concluded that transport by magnetic buoyancy should be considered as a possible mechanism for extra mixing through the radiative zone, as is required by both stellar observations and the extensive isotopic data on circumstellar condensates found in meteorites.
\end{abstract}

Subject headings: MHD — stars: AGB and port-AGB — stars: evolution — stellar dynamics

\section{INTRODUCTION}

Many aspects of the physics and evolution of low-mass stars are not adequately treated in available stellar models. One of these problems is revealed by the photospheric composition of evolved red giants and by presolar dust grains, preserved in meteorites, which are of circumstellar origin. In particular, the isotopic admixture of $\mathrm{CNO}$ and the abundances of a few other species (e.g., $\left.{ }^{7} \mathrm{Li}\right)$ in these environments cannot be reproduced using "first principles" evolutionary codes (see, e.g., Pilachowsky et al. 1993; Grundahl et al. 2002; Wasserburg et al. 2006; Charbonnel \& Do Nascimento 1998). This evidence suggests that mixing mechanisms down to depths just above the $\mathrm{H}$ burning shell must be active during the red giant branch (RGB) and during the asymptotic giant branch (AGB) phases (Gilroy \& Brown 1991; Boothroyd et al. 1994). While abundance problems requiring prolonged mixing already exist on the main sequence, here we are specifically interested in the chemical anomalies found to occur after the "luminosity bump" appears on the RGB and then continuing to occur through the AGB phase. Reviews of these phenomena can be found, e.g., in Kraft (1994) and Charbonnel (2004).

It is now generally agreed that additional transport mechanisms with low mass transfer rates somehow link the convective envelope to stable radiative regions, where some substantial nuclear processing occurs. This requires slow movements of mass to take place inside what is considered as the standard "stable" radiative zone above the $\mathrm{H}$ shell. Parameterized ad hoc calculations have been presented, explaining the above-mentioned chemical and isotopic abundance peculiarities by assuming that material from the convective envelope is brought down, exposed to partial $\mathrm{H}$ burning

\footnotetext{
1 Department of Physics, University of Perugia, via Pascoli, Perugia, 06123, Italy; busso@fisica.unipg.it.

2 The Lunatic Asylum, Division of Geology and Planetary Sciences, California Institute of Technology, Pasadena, CA 91125; gjw@gps.caltech.edu.

3 Physics Division, Argonne National Laboratory, Argonne, IL 60439-4843; nollett@anl.gov.
}

[in the so-called cool bottom processes (CBP)] and then returned through the radiative zone to the convective envelope by some form of weak circulation or diffusion (see Wasserburg et al. 1995; Charbonnel \& Do Nascimento 1998; Nollett et al. 2003; Herwig 2005). The same parameterized scheme devised to account for the above observational data also provides clear explanations for the measurements of high ${ }^{26} \mathrm{Al} /{ }^{27} \mathrm{Al}$ ratios and of distinctive ${ }^{18} \mathrm{O} /{ }^{16} \mathrm{O},{ }^{17} \mathrm{O} /{ }^{16} \mathrm{O}$, and ${ }^{13} \mathrm{C} /{ }^{12} \mathrm{C}$ ratios, in circumstellar grains found in meteorites that are of AGB origin (Choi et al. 1998; Amari et al. 2001; Nittler 2005; Wasserburg et al. 2006). The oxygen data, in particular, require extensive destruction of ${ }^{18} \mathrm{O}$ and enhanced production of ${ }^{17} \mathrm{O}$ (cf. Alexander \& Nittler 1999; Nollett et al. 2003; Clayton \& Nittler 2004). Figures 6, 7, and 16 of Nollett et al. (2003) show clear evidence for the requirements of extra mixing in circumstellar dust grains from AGB stars preserved in meteorites; further evidence from stellar observations is also discussed there. It can be seen from those calculations that to explain the data, processing temperatures $\left(T_{P}\right)$ must be close to that of the H-burning shell, and that the rates of mass transfer must be around $\dot{M} \sim$ $10^{-6} M_{\odot} \mathrm{yr}^{-1}$ (see $\S 3$ below).

Rotation, through shear instabilities and/or meridional circulation, has been suggested as the physical cause of slow deep mixing (Zahn 1992; Denissenkov \& Weiss 1996), although alternative mechanisms have been presented (Denissenkov \& Tout 2003; Charbonnel \& Zahn 2007). Despite formal differences, most models assume that the chemical (and angular momentum) transport has a diffusive nature, with the diffusion coefficient left as a free parameter to match the observations (see, e.g., Denissenkov et al. 1998). A similar approach is commonly used in modeling massive, radiatively stratified stars (see, e.g., Maeder \& Meynet 2004a, $2004 b$, and references therein).

For low-mass red giants, the idea of a purely rotation-induced mixing has recently met difficulties, as the star counterreacts rapidly in such a way that any mixing mechanism is of short duration, and any isotopic change is quenched (Palacios et al. 2006). Similar problems were found by Siess et al. (2004), while looking for 
a rotationally induced formation of the ${ }^{13} \mathrm{C}$ reservoir (often called the ${ }^{13}$ C "pocket"; cf. Busso et al. 1999), which is necessary to produce the $s$ elements in He-burning layers. In contrast, the proposal by Boothroyd et al. (1994) and Wasserburg et al. (1995) of "cool bottom processing" does not specify a driving mechanism, but uses only the path integral of the nuclear reactions and the bulk mass transport rates. This gives, for the appropriate timescale, the resulting chemical and isotopic evolution of the convective envelope for a very wide set of scenarios and provides explicit values for the temperature required to give the appropriate nuclear processing, the corresponding pressure (from the stellar model), and the rates of mass transfer through the processing region. This approach and the stellar models with an adjustable diffusion parameter are essentially equivalent in all results, except that the timescale for transit of processed material to the convective envelope may be much faster for the CBP model.

In a recent paper, Eggleton et al. (2006) reported that mixing might occur due to Rayleigh-Taylor instabilities originating by the inversion in the molecular weight $(\mu)$ induced by ${ }^{3} \mathrm{He}$ burning above the $\mathrm{H}$ shell. Calculations were done by making a local threedimensional hydrodynamical model of the thin layers where $\mu$ decreases, taking the inputs from a one-dimensional stellar code at the RGB phase. The induced mixing appears sufficient to destroy ${ }^{3} \mathrm{He}$ at the surface. Charbonnel \& Zahn (2007) interpreted this mixing event as due to the double diffusive mechanism called the thermohaline instability, early described by Ulrich (1972). They presented a model in which this diffusive process efficiently diminishes ${ }^{3} \mathrm{He}$ in the envelope and gives the required shifts in ${ }^{12} \mathrm{C} /{ }^{13} \mathrm{C}$. The extent to which these recent suggestions are pertinent to the isotopic and chemical shifts on the AGB is still unknown. In our report we will focus on the transport mechanism using the CBP model circulation rates and temperatures and use recent standard stellar structure models to explore the problem.

Because there is no first-principles transport model, we here seek to explore, in a preliminary way, whether the circulation can be accounted for by the buoyancy of magnetic flux tubes that might form in the neighborhood of the nuclear processing region and transport matter upward to the base of the convective envelope. It is likely that differential rotation in such stars can maintain a magnetic dynamo; this problem is very complex, and no fully selfconsistent treatment has yet been found. Certainly, magnetic fields arise by some mechanism.

It has been long established that magnetic flux tubes in a star would provide a buoyant transport mechanism (Parker 1974, 1994) and also that the oscillations of such tubes (Alfvén waves) would evolve into a number of instabilities that could add to the general buoyancy (Tayler 1973; Parker 1974; Spruit 1999, 2002). These phenomena are usually discussed in the context of determining whether they can generate and maintain the dynamo, but this is a complex and unresolved problem that we do not attempt to address.

What we seek to test here is whether magnetic buoyancy phenomena, considered in a zero-order, general treatment, can account for the mass circulation rates inferred from parameterized CBP models. Specifically, we want to establish if they can do so in a model containing toroidal fields with reasonable values of the controlling parameters (magnetic field intensities, buoyancy velocities, fraction of the total radiatively stratified mass that must be involved in the mixing mechanism). Such a simple, order-of-magnitude approach is necessary in order to test whether the development of a detailed MHD model would be of merit.

We shall try to find general guidance on these issues from consideration of solar analogies, as magnetic fields inside AGB stars are essentially unknown. The atmospheres of evolved red giants rotate so slowly that X-ray observations from ROSAT, XMMNewton, and Chandra now exclude the systematic formation of AGB coronae (see, e.g., Ayres et al. 1991; Hünsch et al. 1996). Even in the bright prototype Mira system $o$ Cet, the observed X-ray fluxes $\left(\sim 2 \times 10^{29} \mathrm{erg} \mathrm{s}^{-1}\right)$ cannot be attributed firmly to the AGB star (Soker \& Kastner 2003). However, small magnetic fields (few gauss) have now been shown to be present in the photospheres of evolved red giant stars, with the expected toroidal geometry induced by a dynamo. This was inferred from inspection of circumstellar emission, in particular from $\mathrm{SiO}$ masers formed near the surface (Herpin et al. 2006).

\section{TACHOCLINES IN THE SUN AND IN EVOLVED STARS}

\subsection{The Solar Scenario}

It has been known for more than 80 years (von Zeipel 1924; Eddington 1925) that stars in which energy is transported radiatively cannot rotate as rigid bodies. The centrifugal force from rotation deforms the equipotential surfaces into ellipsoids, while radiation retains spherical symmetry, so radiative and hydrostatic equilibria cannot be maintained simultaneously. This conflict drives meridional circulations known as the Eddington-Sweet effect (Eddington 1925; Sweet 1950). Meridional circulation in a radiatively stratified star implies a transport of angular momentum that drives a state of differential rotation.

According to studies performed by Spruit $(1999,2002)$ on the basis of previous work by Tayler (1973), such a differential rotation in a strongly ionized medium is sufficient to maintain a magnetic dynamo, even in the absence of convection (see also Mestel 1999; Goedbloed \& Poedts 2004). This would generalize the notion of a stellar dynamo as discussed by Parker (1975) for the convective envelope. This specific approach is, however, critiqued in recent papers by Denissenkov \& Pinsonneault (2007) and Zahn et al. (2007).

In the rest of this paper, we do not adopt a specific dynamo model. However, we do assume that toroidal magnetic fields may exist in giant stars, and that buoyant flux tubes will be generated in the radiative layers below the envelope. In fact, whatever the correct model for an effective stellar dynamo might be, it is sufficient for us to know that such a dynamo operates rather ubiquitously in low-mass stars (see e.g., Baliunas \& Vaughan 1985) and to derive from the solar scenario the suggestion that it is generated in radiative layers (see Fan 2004, and discussion below).

Detailed dynamical models for the Sun by Zahn (1992) early suggested a change of about $30 \%$ in the equatorial rotational speed from the inner core to the surface. These models were important anticipations. However, they did not account for a magnetic dynamo and are today no longer quantitatively supported by helioseismology results. These last, in particular after the $\mathrm{SOHO}$ measurements, have established that the convective envelope of the Sun has at its base a region in the nominally radiative zone, roughly $0.04 R_{\odot}$ and $\sim 0.01 M_{\odot}$ thick (called the tachocline) in which rotation gradually passes from rigid (in the central zones) to strongly differential (in the convective outer layers). For the Sun the angular velocity of the core is intermediate between the equatorial and high-latitude surface spin rates. For recent reviews on these subjects see Fan (2004) and Miesch (2005). Models of radiative dynamos, despite the specific problems they may present, have the merit of accounting for helioseismology results (Eggenberger et al. 2005) and of explaining how the rigid rotation of the core can be maintained, despite the contrary arguments by von Zeipel (1924), if the dynamo soaks up the transported angular momentum as quickly as meridional circulation can provide it. 
Observations of the Sun's magnetically active regions, especially at intermediate and low latitudes, are now understood in the framework of an accepted paradigm. The current interpretation (Fan 2004; Miesch 2005) includes the following elements. (1) A dynamo mechanism is generated in the tachocline (a nominally radiative region), maintaining toroidal fields, probably organized as isolated, thin flux tubes (Spruit 1981). (2) The tube profiles are greatly modified into various undulatory shapes (Alfvén waves), sometimes evolving into instabilities (Spruit \& van Ballegooijen 1982). Among them, kink-type modes evolve into $\Omega$-shaped loops with strong radial components (Parker 1974, 1994). (3) Such buoyant loops penetrate the convective layer and then emerge forming the corona; photospheric active regions and sunspots are cross sections of bundles of many flux tubes ("spaghetti model") and are the roots of coronal loops. Magnetic buoyancy is compensated by complex downflows, providing a sort of asymmetric circulation (Spruit 1997). We will assume that a layer analogous to the tachocline exists in RGB and AGB stars, and that flux tubes will rise through this region as they do in the Sun.

In the Sun, magnetic field intensities in flux tubes are of a few $\times 10^{3} \mathrm{G}$ in sunspots, which emerge from the photosphere. These fields must considerably exceed $10^{5} \mathrm{G}$ deep in the radiative tachocline from which they come (Rempel et al. 2000; Schüssler \& Rempel 2002). Certainly, the fields exceed $10^{4} \mathrm{G}$ (Fan 2004) in active regions at the bottom of the convective layer. For the Sun, the value of a few $\times 10^{4} \mathrm{G}$ corresponds to equipartition of energy between the magnetic $\left(B^{2} / 8 \pi\right)$ and convective-kinetic $\left(1 / 2 \rho v_{c}^{2}\right)$ forms in the innermost convective zones. For the typical density of $0.15 \mathrm{~g} \mathrm{~cm}^{-3}$ (Bahcall et al. 2001), this implies that, at equipartition, the convective velocity $v_{c}$ averages tens of meters per second.

The observed values of the solar magnetic flux, which are at the level of a few $\times 10^{21}$ Maxwell in "small" active regions (bundles of many filaments, see e.g., Zwaan 1987, his Table 2), together with local field intensities of a few $\times 10^{4} \mathrm{G}$, imply that magnetized zones of $\sim 1000-2000 \mathrm{~km}$ in radius exist deep in the convective layer. We shall use this estimate of flux tube dimensions in calculations below.

\subsection{Investigating the Links between Magnetic Buoyancy and CBP}

Subadiabatic zones below the convective envelope, qualitatively similar to the solar tachocline (i.e., regions where the heat transport occurs through radiative processes, even in the absence of a strong chemical stratification) can be found in low-mass stars after they have reached the red giant branch, both in the final thermally pulsing phases of the AGB (cf. Nollett et al. 2003) and in preceding RGB stages, which are of much longer duration. The necessary condition is that the advancing $\mathrm{H}$ shell has erased the chemical discontinuities left behind by core $\mathrm{H}$ burning and by the first dredge-up. In all such zones, where the molecular weight gradient is close to zero, stability against matter circulation is not guaranteed (Fricke \& Kippenhahn 1972; see also Collins 1989, chap. 7), and cool bottom processes might occur. Results using the code of Nollett et al. (2003) show that the data can be described by a mass mixing rate of roughly $10^{-6} M_{\odot} \mathrm{yr}^{-1}$ (AGB case) or $4 \times 10^{-8} M_{\odot} \mathrm{yr}^{-1}$ (RGB case) through the top $80 \%-90 \%$ by mass $(97 \%-99 \%$ by radius) of the region between the hydrogen-burning shell and the base of the convective envelope. (These numbers will be developed in more detail in $\S 3$.)

Here we seek to ascertain whether magnetic buoyancy is a plausible option to transport matter in red giants, and to provide the mass circulation rate $\dot{M}$ required by CBP. The requirements from CBP are only a rate of transfer and the maximum temperature of burning. One of the rate-limiting conditions for mass transfer occurs at the boundary between the convective envelope and the radiative zone. The fraction of mass $\left(f_{m}\right)$ at the upper part of the radiative zone made up of flux tubes must be small, so that the stellar structure is not greatly disturbed. The velocity of transport across the boundary must also be compatible with available estimates. We can write the mass flow rate as

$$
\dot{M} \simeq 4 \pi r^{2} \rho c_{s} f_{m} f_{t},
$$

where $c_{s}$ is the velocity of sound, $r$ is the radius at the convective envelope base, $\rho$ is the mass density, and $f_{t}$ is the ratio between the velocity of transport across the boundary to that of sound. Using the local values of the density and of the sound speed obtained from the stellar model, we will first verify that the $\dot{M}$ required for CBP corresponds to small $f_{m}$ and $f_{t}$, so that the fraction of mass at the upper radiative zone interface, which is provided by buoyant flux tubes, is $f_{m} \ll 1$, and that the transport velocity across the boundary $\left(f_{t} \times c_{s}\right)$ is reasonable. We will further check whether the magnetic field requirements of our flux-tube model are consistent with small $f_{m}$ and $f_{t}$.

We can also derive from $\dot{M}$ the rate $\dot{N}_{t}$ at which rising flux tubes must arrive at the convective envelope. At the initial position $r_{0}$ above the $\mathrm{H}$ shell let a flux tube be a torus with an initial radius $a_{0}$, length $l_{0}=2 \pi r_{0}$, and a magnetic field intensity $B_{0}$. The volume of the tube is $2 \pi^{2} r_{0} a_{0}^{2} \mathrm{~cm}^{3}$. At the local density $\rho_{0}$ this corresponds to a mass of $2 \pi^{2} r_{0} \rho_{0} a_{0}^{2}$. During an evolutionary phase of duration $\Delta t_{\mathrm{ph}}$, enough tubes must be deposited into the envelope to guarantee the isotopic changes provided by CBP. In order to achieve this, the convective envelope itself must be mixed, within the available time $\Delta t_{\mathrm{ph}}$, with an amount of H-burning processed material equal to a fraction $\alpha$ of its mass $M_{E}$ (in solar units). The number $N_{t}$ of the tubes must therefore be of the order of $\alpha M_{E}\left(1.989 \times 10^{33} \mathrm{~g}\right) /\left(2 \pi^{2} r_{0} \rho_{0} a_{0}^{2}\right)$. The rate at which these flux tubes must reach the boundary layer is thus

$$
\dot{N}_{t}=\frac{\alpha M_{E}\left(1.989 \times 10^{33} \mathrm{~g}\right)}{2 \pi^{2} r_{0} \rho_{0} a_{0}^{2} \Delta t_{\mathrm{ph}}}=\frac{\dot{M}}{2 \pi^{2} r_{0} \rho_{0} a_{0}^{2}}=\frac{\hat{v}}{\Delta r},
$$

where $\hat{v}$ is the average velocity of the tubes, and $\Delta r$ is the distance from the processing zone to the convective envelope.

We can now explore the implications of the mass circulation requirements on the magnetic fields near the H-burning shell. We assume, after Parker (1974), that there exists a fractional shift $s$ in density due to the magnetic pressure from the matter density $\rho$ outside a flux tube to the matter density $\rho^{\prime}$ within the flux tube. This shift is given by

$$
\frac{\left(\rho-\rho^{\prime}\right)}{\rho}=\frac{\left(B^{2} / 8 \pi\right)}{P} \equiv s .
$$

Applying equation (3) throughout the motion of a flux tube assumes that the interior of the tube is always at the local temperature. This condition is plausible only if the buoyancy velocity is sufficiently small (below few km s${ }^{-1}$; cf. Parker 1974).

Balancing the buoyancy force per unit length on the flux tube $\left[F=\pi a^{2} g(r)\left(\rho-\rho^{\prime}\right)\right]$ with the drag force per unit length $\left(1 / 2 C_{D} \rho a v^{2}\right)$, we obtain $v^{2}=\left(2 \pi / C_{D}\right) g(r) a(r) \Delta \rho / \rho$, where $g(r)$ is the acceleration of gravity at the position $r$, and $C_{D}$ is the aerodynamic drag coefficient. Note that there is little mass in the radiative layer of either the RGB or AGB star, so that $g(r) / g_{0} \sim\left(r_{0} / r\right)^{2}$. Then

$$
v^{2}(r)=\frac{2 \pi a(r) g_{0}}{C_{D}}\left(\frac{r_{0}}{r}\right)^{2} \frac{\Delta \rho}{\rho} .
$$


TABLE 1

Relevant Parameters in the Subconvective Layers

\begin{tabular}{|c|c|c|c|c|c|c|}
\hline Stellar Zone & $M / M_{0}$ & $r / R_{0}$ & $\begin{array}{c}P \\
\left(\text { dyne } \mathrm{cm}^{-2} \text { ) }\right.\end{array}$ & $\begin{array}{c}T \\
(\mathrm{~K})\end{array}$ & $\begin{array}{c}\rho \\
\left(\mathrm{g} \mathrm{cm}^{-3}\right)\end{array}$ & $\begin{array}{c}c_{S} \\
\left(\mathrm{~cm} \mathrm{~s}^{-1}\right)\end{array}$ \\
\hline \multicolumn{7}{|c|}{ (a) AGB phase, ${ }^{\mathrm{a}} M_{0}=1.207 M_{\odot}, M_{E}=0.567 M_{\odot}, R_{0}=331 R_{\odot}, \Delta t_{\mathrm{ph}}=2 \times 10^{6} \mathrm{yr}$} \\
\hline H-shell position & 0.529999 & $6.59 \times 10^{-5}$ & $1.42 \times 10^{17}$ & $6.34 \times 10^{7}$ & 17.71 & $1.07 \times 10^{8}$ \\
\hline Maximum CBP penetration ${ }^{\mathrm{b}}$.......... & 0.530049 & $8.57 \times 10^{-5}$ & $4.24 \times 10^{16}$ & $4.92 \times 10^{7}$ & 4.13 & $1.19 \times 10^{8}$ \\
\hline 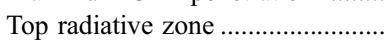 & 0.530519 & $2.26 \times 10^{-3}$ & $1.60 \times 10^{11}$ & $2.3210^{6}$ & $2.9210^{-4}$ & $2.75 \times 10^{7}$ \\
\hline Bottom convective zone ................. & 0.530558 & $2.46 \times 10^{-3}$ & $1.29 \times 10^{11}$ & $2.17 \times 10^{6}$ & $2.48 \times 10^{-4}$ & $2.67 \times 10^{7}$ \\
\hline
\end{tabular}

(b) RGB phase, ${ }^{\mathrm{a}} M_{0}=1.499 M_{\odot}, M_{E}=1.115 M_{\odot}, R_{0}=53 R_{\odot}, \Delta t_{\mathrm{ph}}=4.3 \times 10^{7} \mathrm{yr}$

\begin{tabular}{|c|c|c|c|c|c|c|}
\hline H-shell position. & 0.251564 & $5.41 \times 10^{-4}$ & $1.87 \times 10^{17}$ & $4.19 \times 10^{7}$ & 43.05 & $2.52 \times 10^{8}$ \\
\hline Maximum CBP penetration ${ }^{\mathrm{c}} \ldots \ldots .$. & 0.252093 & $9.34 \times 10^{-4}$ & $1.87 \times 10^{16}$ & $2.51 \times 10^{7}$ & 5.19 & $7.44 \times 10^{7}$ \\
\hline 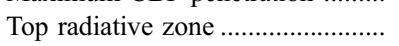 & 0.255296 & $1.58 \times 10^{-2}$ & $1.05 \times 10^{12}$ & $2.42 \times 10^{6}$ & $2.93 \times 10^{-3}$ & $2.32 \times 10^{7}$ \\
\hline Bottom convective zone ................ & 0.255528 & $1.72 \times 10^{-2}$ & $8.49 \times 10^{11}$ & $2.26 \times 10^{6}$ & $2.55 \times 10^{-3}$ & $2.23 \times 10^{7}$ \\
\hline
\end{tabular}

(c) The present Sun, ${ }^{\mathrm{d}} M=M_{\odot}, R=R_{\odot}$

\begin{tabular}{|c|c|c|c|c|c|c|}
\hline Tachocline base. & 0.972383 & 0.6880 & $7.09 \times 10^{13}$ & $2.36 \times 10^{6}$ & 0.219 & $2.45 \times 10^{7}$ \\
\hline 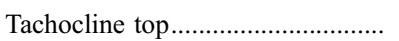 & 0.980219 & 0.7279 & $4.34 \times 10^{13}$ & $2.01 \times 10^{6}$ & 0.155 & $2.18 \times 10^{7}$ \\
\hline Bottom convective zone ................... & 0.980280 & 0.7282 & $4.32 \times 10^{13}$ & $2.00 \times 10^{6}$ & 0.150 & $2.15 \times 10^{7}$ \\
\hline
\end{tabular}

${ }^{\text {a }}$ From a stellar model with initial mass $1.5 M_{\odot}$ and initial metallicity one-half solar.

${ }^{\mathrm{b}}$ Defined as the layer where $\log T=\log T_{P}=\log T_{\mathrm{H}}-0.1$ (Nollett et al. 2003).

${ }^{c}$ Assumed as the layer where $\log T_{P}=7.4$ (Substantial production of ${ }^{13} \mathrm{C}$ ).

d From Bahcall et al. (2001), (data published electronically).

Assuming conservation of magnetic flux $B(r)=B_{0} a_{0}^{2} / a(r)^{2}$ and conservation of mass $a(r)^{2}=\rho_{0} a_{0}^{2} r_{0} /[\rho(r) r]$ within the flux tube, one derives

$$
B(r)=B_{0} \frac{\rho(r) r}{\rho_{0} r_{0}} .
$$

Hence, equation (3) becomes

$$
\frac{\Delta \rho}{\rho}=\left(\frac{B_{0}^{2}}{8 \pi}\right)\left(\frac{r}{r_{0}}\right)^{2}\left(\frac{\rho}{\rho_{0}}\right)^{2} \frac{1}{P}
$$

The velocity can therefore be expressed as

$$
v(r)=\frac{1}{2}\left[\frac{\rho(r)}{\rho_{0}}\right]^{3 / 4}\left(\frac{r}{r_{0}}\right)^{-1 / 4}\left(\frac{g_{0} a_{0}}{C_{D}}\right)^{1 / 2}\left[\frac{B_{0}}{\sqrt{P(r)}}\right]
$$

or

$$
\frac{v(r)}{v_{0}}=\left(\frac{r}{r_{0}}\right)^{-1 / 4}\left[\frac{\rho(r)}{\rho_{0}}\right]^{3 / 4}\left[\frac{P(r)}{P_{0}}\right]^{-1 / 2} .
$$

Concerning the effective (average) velocity of the buoyancy motion over the trajectory, $\hat{v}$, let the time of transport of an individual flux tube across the radiative layer be $t_{T}$; then $\hat{v} t_{T}=\Delta r(\Delta r$ being the distance from the starting position near the $\mathrm{H}$ shell, $r_{0}$, to the base of the convective envelope, $r_{E}$ ). From equation (7a) one can then compute

$$
t_{T}=\int_{r_{0}}^{r_{E}} \frac{d r}{v(r)}
$$

and finally

$$
\hat{v}=\frac{\Delta r}{\int_{r_{0}}^{r_{E}} d r / v(r)} .
$$

The above rough approximations are sufficient for order-ofmagnitude estimates applied to specific physical conditions inside evolved red giant stars. In the $\S 3$, we will estimate $f_{m}, f_{t}$, and $\hat{v}$ from requirements on $\dot{M}$ and $T_{P}$. From $\hat{v}$, we will use equations (7b) and (8) to infer the magnitude of the magnetic field required at the bottom of the CBP circulation in order for buoyant flux tubes to carry it.

\section{REQUIREMENTS ON MAGNETIC BUOYANCY FOR EVOLVED RED GIANTS}

In order to make some quantitative estimates of the magnetic fields that would account for CBP nucleosynthesis, we shall consider, as a reference, the case of a $1.5 M_{\odot}$ red giant, with a metallicity half solar and an internal structure as computed by Straniero et al. (1997) and Busso et al. (2003), including mass loss with the parameterization by Reimers (1975). In this model we shall examine first the AGB situation. Here the occurrence of CBP has quite stringent requirements, as it must affect nuclei up to $\mathrm{Mg}$-Al in a rather short interval of time and must reduce the carbon isotopic ratio ${ }^{12} \mathrm{C} /{ }^{13} \mathrm{C}$ in the envelope in competition with the ongoing, recurrent enhancement of the ${ }^{12} \mathrm{C}$ abundance provided by the third dredge-up. According to Nollett et al. (2003), the whole range of the observations can be accounted for if circulation rates are in the interval of $10^{-7}-10^{-5} M_{\odot} \mathrm{yr}^{-1}$. We choose $\dot{M}=10^{-6} M_{\odot} \mathrm{yr}^{-1}$ as a representative case.

A typical structure of the radiative zone above the H-burning shell, in the AGB phase of our model star of initially $1.5 M_{\odot}$, is illustrated in Table $1 a$. The values given are typical for the interpulse periods (those shown are for the period between the 8th and the 9th occurrence of the third dredge-up). The same physical properties are also plotted in Figure 1. The envelope mass $M_{E}$ and the radius $R_{0}$ of the model star at the phase considered (where mass loss has reduced the total mass to $1.207 M_{\odot}$ ) are shown in the top of Table $1 a$. The total time available for the thermally pulsing AGB stage, summing all interpulse periods (and excluding the relatively short duration of dredge-up episodes) is $\Delta t_{\mathrm{ph}} \simeq 2 \times 10^{6} \mathrm{yr}$. 


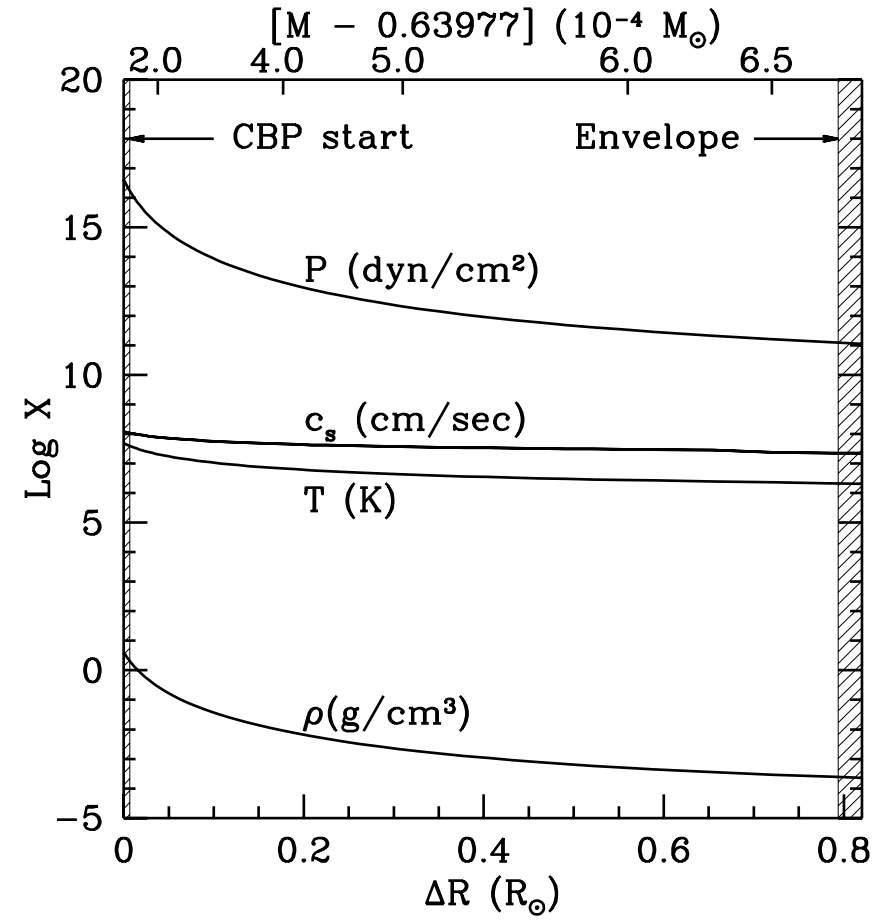

FIG. 1.- Behavior of pressure, density, temperature, and sound speed as a function of the distance (expressed both in radius and in mass) from the point where we assume CBP starts. The plot shows the radiative layers below the convective envelope, for the thermally pulsing AGB phases of a $1.5 M_{\odot}$ star with half solar metallicity (see text).

The depth (temperature) to which the circulating mass must reach is that characterized by $\log T_{P}=\log T_{\mathrm{H}}-0.1$ (Nollett et al. 2003). Here $T_{\mathrm{H}}$ is the temperature of the hydrogen-burning shell, and $T_{P}$ is the temperature at the maximum depth at which matter is transported.

The conditions pertinent to the RGB stages where we consider $\mathrm{CBP}$ correspond to the initial state where the advancing H-burning shell has erased the chemical discontinuities left behind by core $\mathrm{H}$ burning and by the first dredge-up. On the RGB there is no ${ }^{12} \mathrm{C}$ added to the envelope, as occurs instead with the repeated dredgeups on the AGB. An RGB structure used for that stage is shown in Table $1 b$, in the same format as before. The structure is also sketched in Figure 2. The depth to which the circulating mass must reach is taken at $\log T_{P}=7.4$, sufficient for a large fractional change in the ${ }^{13} \mathrm{C}$ content. The total time on the RGB for providing the nuclear processed material is $\Delta t_{\mathrm{ph}}=4.3 \times 10^{7} \mathrm{yr}$.

For the RGB stages, $\mathrm{CNO}$ isotopes (and $\mathrm{Li}$ ) are known to be affected by $\mathrm{CBP}$, the observed ${ }^{12} \mathrm{C} /{ }^{13} \mathrm{C}$ ratio going down from an initial value of $\sim 25$ to about $10-13$ (in Population I stars). The RGB evolution thus provides ${ }^{13} \mathrm{C}$ enrichment and some ${ }^{12} \mathrm{C}$ destruction $(\sim 30 \%)$. Contrary to the AGB case, no concurrent ${ }^{12} \mathrm{C}$ enrichment by dredge-up is present. Moreover, the time available for mixing is much longer than for the AGB, so that we expect much less challenging conditions for the required magnetic fields.

As a comparison, the physical properties for the Sun below the convective envelope are shown in Table $1 c$. Note that in the Sun the tachocline mass is $\Delta M=7.8 \times 10^{-3} M_{\odot}$, and its thickness is $\Delta R=0.04 R_{\odot}$. The mass of the overlying convective envelope is $0.02 M_{\odot}$. In contrast, for the AGB and RGB cases the radiative layer has a mass from a few $\times 10^{-4}$ to a few $\times 10^{-3} M_{\odot}$, and a much larger thickness of $\Delta R \sim 0.79-0.86 R$. . In addition, the mass of the convective envelope is obviously very different from the Sun in these extended red giants, and ranges from 0.5 to more than $1 M_{\odot}$.

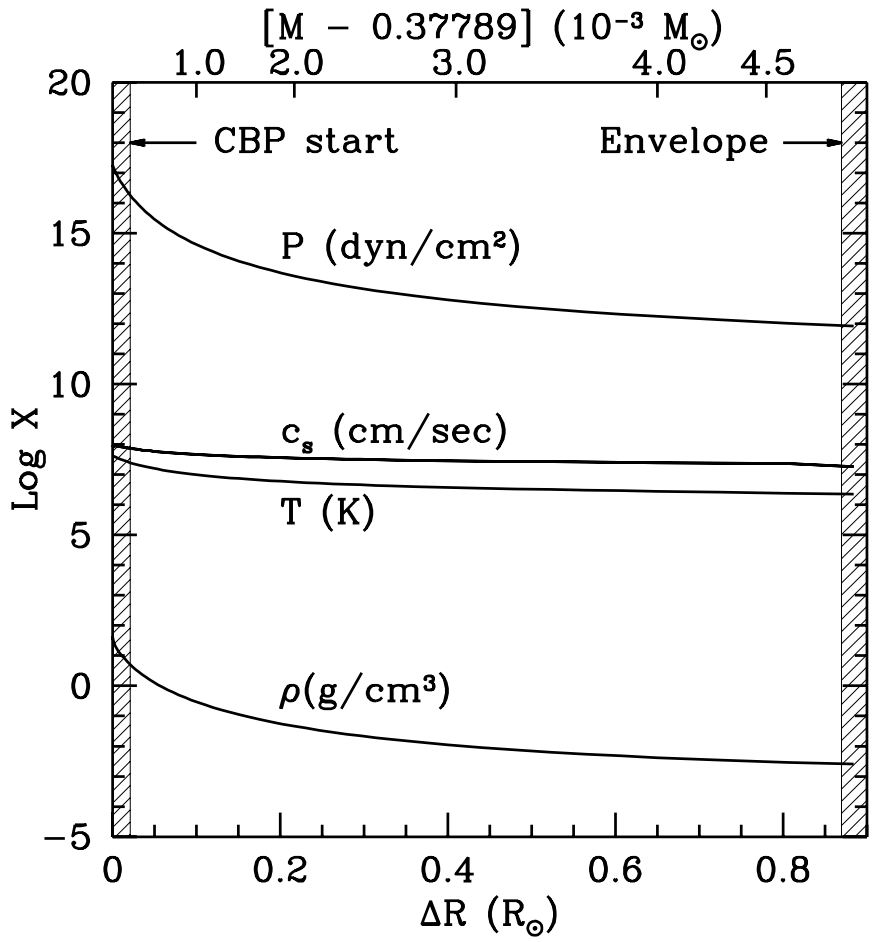

FIG. 2.- Typical structure of the radiative layers below the convective envelope on the RGB, characterizing the stages after the luminosity bump and up to core He ignition (see text).

Thus, the distances traveled by the hypothesized flux tubes in both the RGB and AGB stars is 20 times larger than for the Sun, and the overlying envelopes are also much larger.

\subsection{The $A G B$ Case}

Adopting, at the radiative-convective boundary of the AGB stages, $\dot{M}=10^{-6} M_{\odot} \mathrm{yr}^{-1}$ in equation (1), together with the local density of $2.48 \times 10^{-4} \mathrm{~g} \mathrm{~cm}^{-3}$ and with the local velocity of sound of $2.67 \times 10^{7} \mathrm{~cm} \mathrm{~s}^{-1}$ (Table $1 a$ ), from equation (1) one gets, for material transport across the convective envelope border, $f_{m} f_{t} \simeq 2.33 \times 10^{-7}$.

We do not have an a priori estimate for the buoyancy velocity. Fan (2004) suggests that, near the convective border of the Sun, it can be $v \simeq 10^{-3}|\delta|^{-1} \mathrm{~cm} \mathrm{~s}^{-1}$, where $\delta$ is the difference between the logarithmic thermal gradient $(d \log T / d \log P)$ and the adiabatic one. As this difference becomes typically $\delta \sim-10^{-5}$ in the last subadiabatic layers below the convective solar envelope, the value of $v$ is close to $1 \mathrm{~m} \mathrm{~s}^{-1}$. Values of this order would imply $f_{t} \cong 3.6 \times 10^{-6}$ and $f_{m} \cong 0.07$. As we shall see later (Fig. 3 ), from our formulae the AGB buoyancy velocity at base of the convective envelope turns out to be higher, typically $1 \mathrm{~km} \mathrm{~s}^{-1}$. In this case $f_{t} \cong 3.6 \times 10^{-3}$, and $f_{m} \cong 7 \times 10^{-5}$. Thus, in any case only a small fraction of the mass in the top of the radiative zone must be from flux tubes.

According to Nollett et al. (2003), CBP must connect the envelope with internal zones where the maximum temperature $T_{P}$ is as high as $\log T_{P}-\log T_{\mathrm{H}}=-0.1$. Transport from this level into the convective envelope provides an adequate mass of processed material if $\dot{M} \sim 10^{-6} M_{\odot} \mathrm{yr}^{-1}$ and $\alpha \simeq 1$.

For the AGB case shown in Table $1 a$, and assuming the flux tubes to be toroidal at all $r$, the ratio $r_{0} / r$ between the innermost region where CBP must penetrate and the base of the envelope is $\sim 1 / 28.7$. The density ratio is $\rho_{0} / \rho \sim 1.66 \times 10^{4}$. This implies that $a_{0} / a \sim 1 / 24$. If we assume that the flux tubes have the same size 


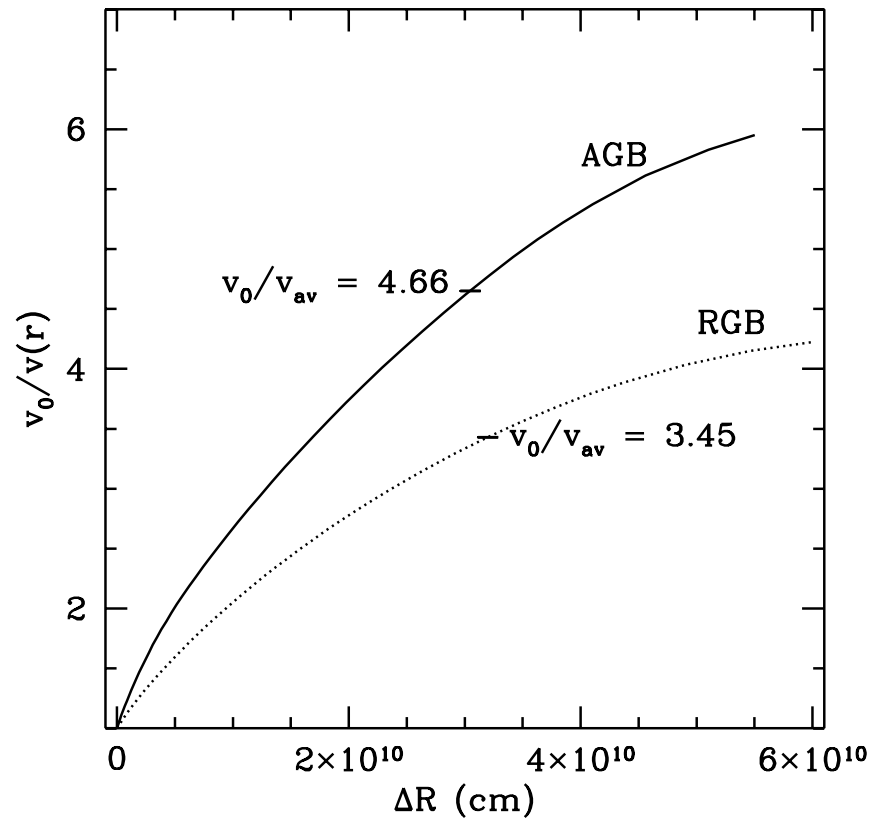

FIG. 3.-Buoyancy velocity - expressed, as in eq. (7b), through the ratio between the maximum value and the local value at any $r$ - as a function of the distance from the point where we assume CBP starts. The ratio of the initial velocity and the average velocity is shown for the two cases (see text).

of $\sim 1000-2000 \mathrm{~km}$ at the base of the convective envelope as in the Sun (as discussed in $\S 2.1$ ), we obtain an initial tube radius of $a_{0} \sim 42-83 \mathrm{~km}$ in the zone where CBP starts. We adopt $a_{0}=65 \mathrm{~km}$ for illustration. For an envelope mass of $M_{E}=0.567 M_{\odot}$, and with the parameters of Table $1 a$, equation (2) yields $\dot{N}_{t} \simeq 2.6 \times$ $10^{-6} \mathrm{~s}^{-1}$ ( $\sim 7$ per month). The corresponding average velocity to deliver a parcel of matter to the envelope (at $\left.r=5.7 \times 10^{10} \mathrm{~cm}\right)$ is $\hat{v} \sim 1.45 \mathrm{~km} \mathrm{~s}^{-1}$ if only one flux tube rises at a time. This relatively low velocity justifies a posteriori our assumption of thermal equilibrium between the magnetized flux tube and the environment. If any form of magnetic diffusion or phase mixing (Spruit 1999) were to occur, then a fraction of the flux tubes originally formed would not reach the convective zone interface, so the net rate of generation of flux tubes would have to be greater. If many ( $n$ ) flux tubes form in the processing region at essentially the same time, then the velocity is decreased by a factor $n$. The number of flux tubes that can be generated simultaneously just above the $\mathrm{H}$ shell is not known. Insofar as this number is not much greater than unity, the conclusions are not strongly dependent on $n$. In all the following discussion in this paper we shall take $n=1$. The common modeling scheme called the "thin flux tube approximation" (Schüssler 1977; Spruit 1981), which we have followed, essentially assumes that flux tubes remain as individual entities deep in the stellar interiors. We note in contrast that the magnetized zones in the solar envelope to which we make reference in deriving $a_{0}$ are bundles of many individual filaments. We cannot ascertain now whether these bundles are formed as groups of separate filaments or as individual flux tubes, but we note that turbulence in the envelope is in itself a very likely and efficient cause for locally shredding magnetic tubes into many perturbed filaments (cf. Stothers 2004).

If the average velocity necessary to provide processed matter to the envelope at the required rate is of the order estimated above, then we can derive the value $v_{0}$ of the maximum velocity (the one pertaining to the innermost layers, near the $\mathrm{H}$ shell). For the AGB, using equations (8) and (7a), and numerically computing the integrand gives $v_{0} \sim 6.8 \mathrm{~km} \mathrm{~s}^{-1}$ for $\hat{v}=1.45 \mathrm{~km} \mathrm{~s}^{-1}$ : the function of equation ( $7 \mathrm{~b}$ ) is shown in Figure 3 (solid line). Using the pressure of $4.24 \times 10^{16}$ dyne $^{-2}$ in the CBP starting zone, we obtain a magnetic field value in the innermost layers of $B_{0} \simeq 2.3 \times 10^{7} C_{D}^{1 / 2}$.

The value of $C_{D}$ is uncertain. At high Reynolds numbers it is usually assumed to be close to unity, after Batchelor (1967), but the range of the possible values might extend down to about 0.04 (H. Hornung 2007, private communication), if one considers that the boundary between the flux tubes and the surrounding medium permits slip. The lowest necessary fields would therefore correspond to $C_{D}^{1 / 2}=0.20$. We can note that the stored fields in the solar radiative layers do not largely exceed the equipartition value (Moreno Insertis 1986). If equipartition is applicable for AGB stars, the condition $B_{0}^{2} / 8 \pi=1 / 2 \rho_{0} v_{0}^{2}$ and the velocity $v_{0}$ give $B_{0}=5 \times 10^{6} \mathrm{G}$. This formally corresponds to a value of $C_{D}=$ 0.05 . As $C_{D}$ appears under a square root, changing its value has a moderate effect on the field (for example doubling the above choice, i.e., adopting $C_{D}=0.1$, would imply a field $B_{0}=7 \times 10^{6} \mathrm{G}$, still rather close to equipartition).

With due consideration for the uncertainties in the above calculations, it is therefore clear that strong fields are needed near the $\mathrm{H}$ shell to drive CBP, at the level of several megagauss (MG) in the innermost zones reached by mass circulation. At the base of the convective envelope, using equation (5) and $B_{0}=5 \times 10^{6} \mathrm{G}$ we get $B_{E} \simeq 9 \times 10^{3} \mathrm{G}$. This is similar to the fields found in the deep layers of the solar convective envelope.

\subsection{The RGB Case}

Unambiguous evidence for CBP in first-ascent red giants is offered by the ratio ${ }^{12} \mathrm{C} /{ }^{13} \mathrm{C}$, which decreases more sharply than can be accounted for by first dredge-up. Average values of $\dot{M}$ and $T_{P}$ suitable for the RGB can then be estimated from inspection of the stellar model and from simple considerations. We refer to late RGB stages, after the $\mathrm{H}$ shell has erased previously established chemical discontinuities. From this moment on, the layers below the convective envelope have a rather homogeneous composition, so that the chemical stratification does not seriously hamper matter circulation. In the model of Table $1 b$, the duration of this phase is about $4.3 \times 10^{7} \mathrm{yr}$. The convective envelope mass decreases over this time period from 1.203 to $1.001 M_{\odot}$, with an average value of $M_{E}=1.11 M_{\odot} . \mathrm{CBP}$ must bring the ${ }^{12} \mathrm{C} /{ }^{13} \mathrm{C}$ ratio of this convective envelope down to $\sim 10-13$, starting from the typical value of 25 left behind by first dredge-up. This must be obtained by mixing the envelope with a total mass $M_{c}$ of circulated material greatly enriched in ${ }^{13} \mathrm{C}$ ( possibly approaching the $\mathrm{CN}$ cycle equilibrium of ${ }^{12} \mathrm{C} /{ }^{13} \mathrm{C} \lesssim 4$ ). This estimate implies $\alpha \sim 1$, and therefore $\dot{M}$ values larger than $10^{-8} M_{\odot} \mathrm{yr}^{-1}$, over the time interval available. A more detailed calculation of the evolution of the envelope for this RGB star is shown in Figure 4 for $\log T_{P}=7.4$, corresponding to $\log \left(T_{P} / T_{\mathrm{H}}\right)=-0.22$, and $\dot{M}$ in the range of $4 \times 10^{-9}-4 \times 10^{-7} M_{\odot} \mathrm{yr}^{-1}$. These calculations were carried out using the CBP code of Nollett et al. (2003). It can be seen, from the final carbon isotopic ratio obtained, that the estimated value of $\dot{M}$ is correct. As an average, for the RGB case we adopt $\dot{M}=$ $4 \times 10^{-8} M_{\odot} \mathrm{yr}^{-1}$ and $\log T_{P}=7.4$. Note that for this choice the $\mathrm{N} / \mathrm{O}$ ratio is unaffected. ( ${ }^{7} \mathrm{Li}$, not shown in the figure, is instead extensively destroyed).

First we note that the condition established in equation (1), that $f_{t}$ and $f_{m}$ be small, is well fulfilled. Their product is $f_{t} \times f_{m}=$ $8.7 \times 10^{-10}$. For a transport velocity of $\sim 100 \mathrm{~cm} \mathrm{~s}^{-1}$ near the envelope base (Fan 2004 and $\S 3.1$ above), this gives $f_{m} \cong 2 \times 10^{-4}$. As we shall see, by estimating the buoyancy velocity at the envelope base through equation (7b) one gets a higher value of $\sim 10 \mathrm{~m} \mathrm{~s}^{-1}$ (Fig. 3). In this case $f_{m} \cong 2 \times 10^{-5}$. Again, for any reasonable choice of the transport velocity, only a minimal fraction 


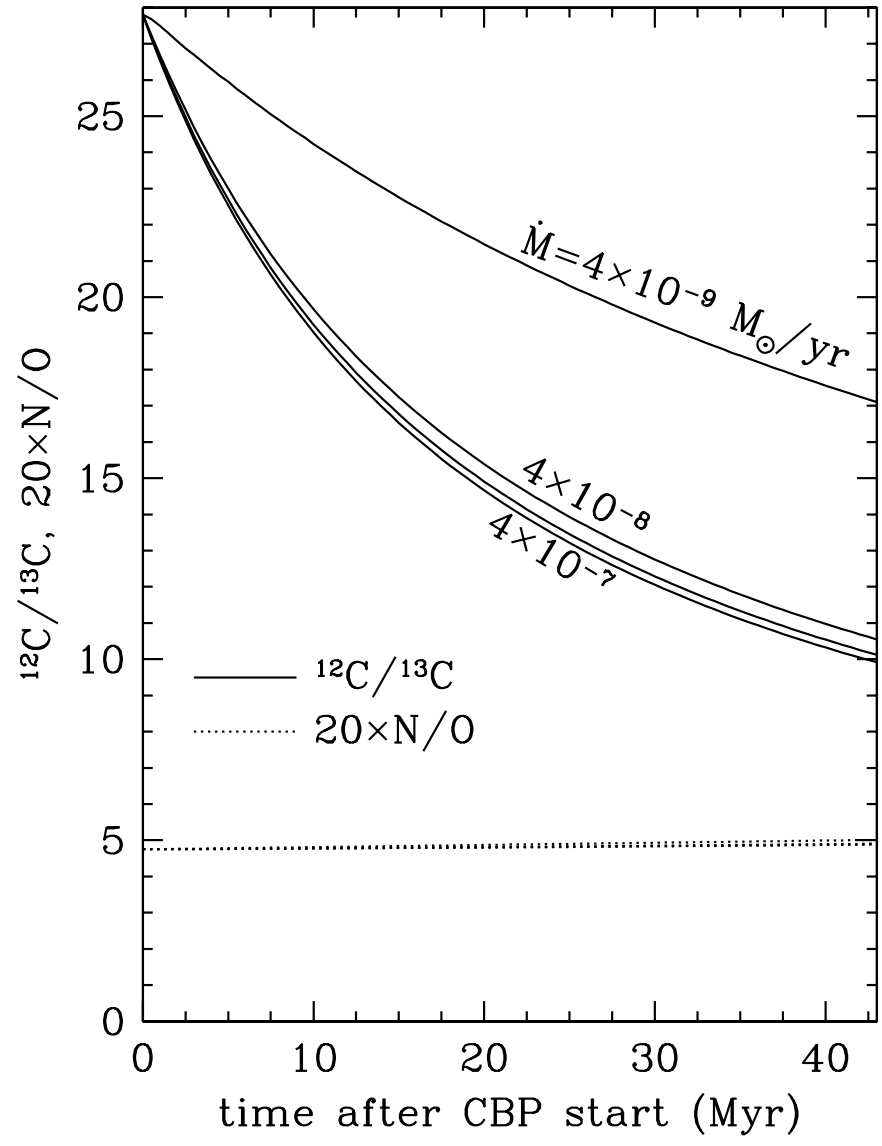

Fig. 4.-CBP model results for the RGB stellar structure of Fig. 2, four different mixing rates $\dot{M}$, and an assumed maximum mixing depth with $\log \left(T_{P} / T_{\mathrm{H}}\right)=$ -0.22 . Curves show ${ }^{12} \mathrm{C} /{ }^{13} \mathrm{C}$ and $\mathrm{N} / \mathrm{O}$ as functions of time after start of $\mathrm{CBP}$. Three values of $\dot{M}$ are shown next to the corresponding ${ }^{12} \mathrm{C} /{ }^{13} \mathrm{C}$ curves; the fourth is $10^{-7} M_{\odot} \mathrm{yr}^{-1}$. For this mixing depth, ${ }^{12} \mathrm{C} /{ }^{13} \mathrm{C}$ constraints are satisfied as long as $\dot{M} \gtrsim 10^{-8} M_{\odot} \mathrm{yr}^{-1}$. All four curves for N/O lie on top of each other. Higher $T_{P}$ would require $\dot{M}$ near $4 \times 10^{-8} M_{\odot} \mathrm{yr}^{-1}$ to satisfy the ${ }^{12} \mathrm{C} /{ }^{13} \mathrm{C}$ constraints and would show $\mathrm{N}$ production.

of the mass needs to be from flux tubes at the radiative-convective boundary.

We may now directly obtain the results for the RGB as compared to the AGB. The condition for the average velocity (eq. [2]) applies both to the AGB and to the RGB case. Hence, indicating with subscripts (A) and (R) the AGB and RGB cases, respectively,

$$
\frac{\hat{v}_{\mathrm{R}}}{\hat{v}_{\mathrm{A}}}=\frac{\Delta t_{\mathrm{ph}, \mathrm{A}} \Delta r_{\mathrm{R}}\left(\alpha M_{E}\right)_{\mathrm{R}} r_{0, \mathrm{~A}} \rho_{0, \mathrm{~A}}}{\Delta t_{\mathrm{ph}, \mathrm{R}} \Delta r_{\mathrm{A}}\left(\alpha M_{E}\right)_{\mathrm{A}} r_{0, \mathrm{R}} \rho_{0, \mathrm{R}}}\left(\frac{a_{0, \mathrm{~A}}}{a_{0, \mathrm{R}}}\right)^{2},
$$

with $\alpha_{\mathrm{R}}=1.48$ from $\dot{M}=4 \times 10^{-8} M_{\odot} \mathrm{yr}^{-1}$ as found in the CBP calculation for the RGB. This yields

$$
\frac{\hat{v}_{\mathrm{R}}}{\hat{v}_{\mathrm{A}}}=6.78 \times 10^{-2}\left(\frac{a_{0, \mathrm{~A}}}{a_{0, \mathrm{R}}}\right)^{2} .
$$

A similar relation (derived from eq. [7a]) holds for the maximum velocity $\left[v_{0}=1 / 2\left(g_{0} a_{0} / C_{D}\right)^{1 / 2}\left(B_{0} / P_{0}^{1 / 2}\right)\right]$, so that if $C_{D}$ is equal in both cases

$$
\frac{v_{0, \mathrm{R}}}{v_{0, \mathrm{~A}}}=\frac{\left(g_{0} a_{0}\right)_{\mathrm{R}}^{1 / 2} B_{0, \mathrm{R}} P_{0, \mathrm{~A}}^{1 / 2}}{\left(g_{0} a_{0}\right)_{\mathrm{A}}^{1 / 2} B_{0, \mathrm{~A}} P_{0, \mathrm{R}}^{1 / 2}} .
$$

\begin{tabular}{|c|c|c|c|}
\hline Parameter & AGB & RGB-1 & RGB-2 \\
\hline$a_{0}(\mathrm{~km})$ & 65 & 65 & 150 \\
\hline$\hat{v}\left(\mathrm{~km} \mathrm{~s}^{-1}\right) \ldots \ldots \ldots \ldots \ldots$ & 1.45 & 0.098 & 0.018 \\
\hline$v_{0}\left(\mathrm{~km} \mathrm{~s}^{-1}\right) \ldots \ldots \ldots \ldots \ldots$ & 6.80 & 0.336 & 0.062 \\
\hline$B_{0}(\mathrm{G})$ & $5 \times 10^{6}$ & $3.8 \times 10^{5}$ & $4.8 \times 10^{4}$ \\
\hline$B_{E}(\mathrm{G}) \ldots \ldots \ldots$ & 8600 & 3450 & 440 \\
\hline
\end{tabular}

TABLE 2

Parameters of the Flux Tubes for the Cases Considered

Taking the ratio of equations (10) and (11), together with the relationship between $\hat{v}$ and $v_{0}$ shown in Figure 3 , and using the stellar code parameters of Table $1(a, b)$ we obtain the scaling law

$$
\frac{B_{0, \mathrm{R}}}{B_{0, \mathrm{~A}}}=0.075\left(\frac{a_{0, \mathrm{~A}}}{a_{0, \mathrm{R}}}\right)^{5 / 2}
$$

It is evident that, for the same initial flux tube dimensions, $\left(B_{0, \mathrm{R}} / B_{0, \mathrm{~A}}\right) \lesssim 1 / 10$. The $5 \times 10^{6} \mathrm{G}$ field for the AGB stages would in particular correspond to a field of $\sim 3.8 \times 10^{5} \mathrm{G}$ for the RGB stages. From equations (10) and (2), this corresponds to $\hat{v}=$ $98 \mathrm{~m} \mathrm{~s}^{-1}$ and $\dot{N}_{t}=1.63 \times 10^{-7} \mathrm{~s}^{-1}(\sim 5$ times per year $)$ during the RGB phase.

If we assume instead that $a$ at the bottom of the convective envelope is the same (1000-2000 km) on the RGB as we assumed for the AGB, then the parameters of Table $1 b$ give $a_{0} \sim$ $100-200 \mathrm{~km}$, for an average of $\sim 150 \mathrm{~km}$. Since $\dot{M}_{\mathrm{RGB}}=4 \times$ $10^{-8} M_{\odot} \mathrm{yr}^{-1}$, the rate of flux tube generation for this case, expressed by equation (2), is $\dot{N}_{t}=3 \times 10^{-8} \mathrm{~s}^{-1}$ ( $\sim$ once per year), and the velocities are $\hat{v} \sim 18 \mathrm{~m} \mathrm{~s}^{-1}$ and $v_{0} \sim 62.4 \mathrm{~m} \mathrm{~s}^{-1}$. Using these values of the velocity and $C_{D} \sim 0.05$ to infer an estimate for $B$ in the deepest layers affected by CBP (eq. [7a]), we get $B_{0} \sim$ $4.8 \times 10^{4} \mathrm{G}$, close to the equipartition value $\left(4.3 \times 10^{4} \mathrm{G}\right)$. (Note that the same values for the velocities and the magnetic field $B_{0}$ can also be derived by equations [10], [11], and [12], adopting $a_{0}=150 \mathrm{~km}$ ). The estimate of $B_{0}=4.8 \times 10^{4} \mathrm{G}$ gives $B \sim 440 \mathrm{G}$ at the bottom of the convective envelope from flux conservation.

The above results for the AGB and RGB fields and buoyancy velocities are summarized in Table 2 . Velocities in all cases are small enough that thermal equilibrium with the environment should be achieved. There is a significant difference in the pressure scale height (hence in any scale height for mixing) at the CBP position for the RGB as compared to the AGB. Hence, arguments by Vishniac (1995), in particular his equation (61), would indicate that the larger value of $a_{0}$ in the case RGB-2 of Table 2 may be the correct one for the RGB. For either value of $a_{0}$, the result is that substantial magnetic fields are also required by $\mathrm{CBP}$ on the RGB, although at far lower levels than for the AGB case.

\section{CONCLUSIONS}

We have investigated the consequences resulting from the assumption that buoyant magnetic flux tubes may be responsible for the transport of matter processed in the neighborhood of the hydrogen-burning shell. Such an assumption is not unrealistic, not only on the basis of the solar scenario, but also because the different characteristics of stellar dynamo processes have been recently suggested, on observational grounds, to be linked to the establishment of deep mixing (Böhm-Vitense 2007).

It is assumed that both RGB and AGB stars have dynamos producing toroidal magnetic flux tubes in that region. From the 
observational requirements that matter has undergone nuclear processing and has been transported through the radiative zone to the convective envelope, we know the mass flow rates $(\dot{M})$ that are needed and the temperature of processing $\left(T_{P}\right)$. Furthermore, the structure and evolutionary timescales for both the RGB and AGB stars are known from well-established stellar models. Since evidence for chemical peculiarities induced by CBP is shown only by relatively low-mass red giants ( below $2-3 M_{\odot}$ ), our calculations were done for a star of initial mass $1.5 M_{\odot}$, with half solar metallicity. Assuming transport by buoyant magnetic flux tubes, it was possible to calculate the magnetic fields $B_{0}$ required at the processing zone to give the necessary mass transfer rates. It is shown that to supply the processed material to the AGB star requires large magnetic fields near the $\mathrm{H}$ shell, at the level $B_{0} \sim$ $5 \times 10^{6} \mathrm{G}$ at the bottom of the $\mathrm{CBP}$ region and $B_{E} \sim 9 \times 10^{3} \mathrm{G}$ at the base of the convective envelope. For the RGB case, it was found that the required fields are much smaller, with $B_{0}$ in the range of $5 \times 10^{4}-4 \times 10^{5} \mathrm{G}$ and with $B_{E}$ in the range of $450-3500 \mathrm{G}$, depending on how the flux tube size was estimated. This processing and transport will produce ${ }^{12} \mathrm{C} /{ }^{13} \mathrm{C}$ ratios of $10-12$ in the envelope at the end of the RGB evolution and would extensively destroy Li.

It follows that, if magnetic buoyancy is the means of transport for CBP, then very high fields are required at great depths for the AGB phase and substantial, but much more modest, fields are required for the RGB phase.

There is no direct observational evidence of strong magnetic fields in either RGB or AGB stars. However, it is quite plausible that the rather modest fields we find at the base of the envelope would be greatly subdued when engulfed in the very massive overlying convecting stellar envelope. A rough estimate for AGB stars, using flux conservation, gives surface fields of $\lesssim 20 \mathrm{G}$, in line with observational estimates by Herpin et al. (2006). This is compatible with the fact that AGB stars are not observed to have X-ray emitting coronae. For the RGB case the surface fields would again be small, in the range from $\lesssim 1$ to a few $\mathrm{G}$.

With regard to the high fields found to be necessary for the AGB case, we note that plasma jets of magnetic origin have been observed for some planetary nebulae (cf. Kastner et al. 2003). A planetary nebula is the result of the end of AGB evolution, when the envelope is blown off, leaving a white dwarf remnant. Blackman et al. (2001) considered the development of dynamos in AGB stars as the origin of magnetic fields shaping planetary nebulae. In their calculations they inferred fields of $\sim 5 \times 10^{4} \mathrm{G}$ at the base of the AGB convective envelope (at a radius of $\sim 1.5 R_{\odot}$ ) in order to explain the collimation. $B$ values of up to a few $\times 10^{6} \mathrm{G}$ were inferred in case of low filling factors (which is in fact our case due to the low values of $f_{m}$ ). There is thus independent evidence for large internal magnetic fields ${ }^{4}$ in AGB stars.

It is further of note that white dwarfs, which are the end product of AGB evolution, do sometimes show very high fields. Recent studies have revived the hypothesis that these fields might be fossil remnants of the stellar ones ( Tout et al. 2004; Wickramasinghe \& Ferrario 2005; Ferrario \& Wickramasinghe 2005; Valyavin et al. 2006). Of special importance for this hypothesis is the identification of magnetic configurations, with toroidal components, that can remain stable for extremely long periods (Braithwaite \& Spruit 2006). Many white dwarfs either do not show magnetic fields, or show them at the $\mathrm{kG}$ level; nevertheless fields larger than $1 \mathrm{MG}$, and up to $2 \mathrm{GG}$, are present in $10 \%-20 \%$ of the available white dwarf sample (Liebert et al. 2003; Schmidt et al. 2003; Jordan et al. 2007). Most supermagnetic objects seem to be of relatively high mass $\left(\sim 0.9 M_{\odot}\right)$, but systems with $B=1-100 \times 10^{6} \mathrm{G}$ and with masses $M \leq 0.75 M_{\odot}$ are not infrequent (cf. Liebert et al. 2003, their Tables $1-3$ ). These observations suggest that the high fields of such white dwarfs might be inherited from the AGB precursor, with high enhancement factors ensuing upon the expulsion of the envelope.

From the arguments presented in this paper we conclude that magnetic buoyancy is a very plausible mechanism for transporting material from near the $\mathrm{H}$ shell into the convective envelope for both RGB and AGB stars. This requires very high internal fields for AGB stars with extensive CBP. In our presentation we have only given phenomenological arguments regarding the transport, assuming that the stars provide the required magnetic fields. It is our hope that the broader considerations presented here may stimulate intensive MHD modeling of low-mass stars with very extended envelopes.

The authors would like to thank Hans Hornung for views on the possible values of $C_{D}$. M. B. and A. C. acknowledge support from MURST under contract PRIN2006-022731. G. J. W. acknowledges the support of DOE-FG03-88ER13851 and the generosity of the Epsilon foundation and Caltech's contribution 9178(1123). K. M. N. is supported by the US Department of Energy, Office of Nuclear Physics under contract. DE-AC02-06CH11357. The authors would like to thank the reviewer (clearly an expert on MHD) for a most constructive and useful critical review. We would like to dedicate this paper to Gene Parker and Bob Leighton for their pioneering solar activities.

\footnotetext{
${ }^{4}$ For an update of these calculations see the recent work by Nordhaus et al. (2007), where it is made clear that, in order to maintain the dynamo, a mechanism for supplying energy from the convective motions to the differential rotation is needed, as in the Sun.
}

\section{REFERENCES}

Alexander, C. M. O’D, \& Nittler, L. R. 1999, ApJ, 519, 222

Charbonnel, C., \& Zahn, J.-P. 2007, A\&A, 467, L15

Amari, S., Nittler, L. R., Zinner, E., Gallino, R., Lugaro, M., \& Lewis, R. S. 2001, ApJ, 546, 248

Ayres, T. R., Fleming, T. A., \& Schmitt, J. H. M. M. 1991, ApJ, 376, L45

Bahcall, J. N., Pinsonneault, M. H., \& Basu, S. 2001, ApJ, 555, 990

Baliunas, S. L., \& Vaughan, A. H. 1985, ARA\&A, 23, 379

Batchelor, G. K. 1967, An Introduction to Fluid Dynamics (Cambridge: Cambridge Univ. Press)

Blackman, E. G., Frank, A., Markiel, J. A., Thomas, J. H., \& Van Horn, H. M. 2001, Nature, 409, 485

Böhm-Vitense, E. 2007, ApJ, 657, 486

Boothroyd, A. I., Sackmann, I.-J., \& Wasserburg, G. J. 1994, ApJ, 430, L77

Braithwaite, J., \& Spruit, H. C. 2006, A\&A, 450, 1097

Busso, M., Gallino, R., \& Wasserburg, G. J. 1999, ARA\&A, 37, 239 2003, Publ. Astron. Soc. Australia, 20, 356

Charbonnel, C. 2004, in Origin and Evolution of the Elements, ed. A. McWilliams \& M. Rauch (Pasadena: Carnegie Obs.)

Charbonnel, C., \& Do Nascimento, J. D., Jr. 1998, A\&A, 336, 915

Choi, B.-G., Huss, G. R., Wasserburg, G. J., \& Gallino, R. 1998, Science, 282, 1284

Clayton, D. D., \& Nittler, L. R. 2004, ARA\&A, 42, 39

Collins, G. W., II 1989, The Fundamentals of Stellar Astrophysics (New York:

W. H. Freeman)

Denissenkov, P. A., Da Costa, G. S., Norris, \& J. E., \& Weiss, A. 1998, A\&A, 333,926

Denissenkov, P. A., \& Pinsonneault, M. 2007, ApJ, 655, 1157

Denissenkov, P. A., \& Tout, C. A 2003, MNRAS, 340, 722

Denissenkov, P. A., \& Weiss, A. 1996, A\&A, 308, 773

Eddington, A. S. 1925, Observatory, 48, 73

Eggenberger, P., Maeder, A., \& Meynet, G. 2005, A\&A, 440, L9

Eggleton, P. P., Dearborn, D. S. P., \& Lattanzio, J. C. 2006, Science, 314, 1580

Fan, Y. 2004, Living Rev. Sol. Phys., 1, 1

Ferrario, L., \& Wickramasinghe, D. T. 2005, MNRAS, 356, 615

Fricke, K. J., \& Kippenhan, R. 1972, ARA\&A, 10, 45

Gilroy, K. K., \& Brown, J. A. 1991, ApJ, 371, 578 
Goedbloed, J. P. H., \& Poedts, S. 2004, Principles of Magnetohydrodynamics (Cambridge: Cambridge Univ. Press)

Grundahl, F., Briley, M., Nissen, P. E., \& Feltzing, S. 2002, A\&A, 395, 481

Herpin, F., et al. 2006, A\&A, 450, 667

Herwig, F. 2005, ARA\&A, 43, 435

Hünsch, M., Schmitt, J. H. M. M., Schröder, K.-P., \& Reimers, D. 1996, A\&A, 310,801

Jordan, S., et al. 2007, A\&A, 462, 1097

Kastner, J. H., et al. 2003, ApJ, 591, L37

Kraft, R. P. 1994, PASP, 106, 553

Liebert, J., Bergeron, P., \& Holberg, J. B. 2003, AJ, 125, 348

Maeder, A., \& Meynet, G. 2004a, A\&A, 416, 1023 2004b, A\&A, 422, 225

Mestel, L. 1999, Stellar Magnetism (Oxford: Oxford Univ. Press)

Miesch, M. S. 2005, Living Rev. Sol. Phys., 2, 1

Moreno-Insertis, F. 1986, A\&A, 166, 291

Nittler, L. R. 2005, ApJ, 618, 281

Nollett, K. M., Busso, M., \& Wasserburg, G. J. 2003, ApJ, 582, 1036

Nordhaus, J., Blackman, E. G., Frank, A. 2007, MNRAS, 376, 599

Palacios, A., Charbonnel, C., Talon, S., \& Siess, L. 2006, A\&A, 453, 261

Parker, E. N. 1974, Ap\&SS, 31, 261 1975, ApJ, 198, 205 1994, ApJ, 433, 867

Pilachowski, C. A., Sneden, C., \& Booth, J. 1993, ApJ, 407, 699

Reimers, D. 1975, in Problems in Stellar Atmospheres and Envelopes, ed. B. Baschek, et al. (Berlin: Springer), 229

Rempel, M., Schüssler, M, \& Tóth, G. 2000, A\&A, 363, 789
Schmidt, G. D., et al. 2003, ApJ, 595, 1101

Schüssler, M. 1977, A\&A, 56, 439

Schüssler, M., \& Rempel, M. 2002, in From Solar Min to Max: Half a Solar Cycle with $\mathrm{SOHO}$, ed. A. Wilson (ESA SP-508; Noordwijk: ESA), 499

Siess, L., Goriely, S., \& Langer, N. 2004, A\&A, 415, 1089

Soker, N., \& Kastner, J. H. 2003, ApJ, 592, 498

Spruit, H. C. 1981, A\&A, 98, 155

- 1997, Mem. Soc. Astron. Italiana, 68, 397

1999, A\&A, 349, 189

2002, A\&A, 381, 923

Spruit, H. C., \& van Ballegooijen, A. A. 1982, A\&A, 106, 58

Stothers, R. B. 2004, ApJ, 607, 999

Straniero, O., et al. 1997, ApJ, 478, 332

Sweet, P. A. 1950, MNRAS, 110, 548

Tayler, R. J. 1973, MNRAS, 161, 365

Tout, C. A., Wickramasinghe, D. T., \& Ferrario, L. 2004, MNRAS, 355, L13 Ulrich, R. K. 1972, ApJ, 172, 165

Valyavin, G., et al. 2006, ApJ, 648, 559

Vishniac, E. T. 1995, ApJ, 446, 724

von Zeipel, H. 1924, MNRAS, 84, 684

Wasserburg, G. J., Boothroyd, A. I., \& Sackmann, I.-J. 1995, ApJ, 447, L37

Wasserburg, G. J., Busso, M., Gallino, R., \& Nollett, K. M. 2006, Nucl. Phys. A., 777,5

Wickramasinghe, D. T., \& Ferrario, L. 2005, MNRAS, 356, 1576

Zahn, J.-P. 1992, A\&A, 265, 115

Zahn, J.-P., Brun, A. S., \& Mathis, S. 2007, A\&A, 474, 145

Zwaan, C. 1987, ARA\&A, 25, 83 